\title{
Crawling towards Building Advance Search Engine
}

\author{
Aparna Hambarde ${ }^{1}$, Shwetal Yadav², Aishwarya Dhekane ${ }^{3}$, Dipali Yadav ${ }^{4}$, Mayuri Pawar $^{5}$ \\ Professor, Computer Dept, KJ College, Pune, India ${ }^{1}$ \\ Student, Computer Dept, KJ College, Pune, India ${ }^{2,3,4,5}$
}

\begin{abstract}
In these days situation World Wide internet is flooded with large quantity of knowledge. Finding helpful information from the online is sort of difficult task. Their part several search engines accessible within the market which will solve our purpose. But among all, choosing correct program with extremely effective internet crawler is sort of necessary. There unit several challenges within the style of high performances internet crawler find it irresistible should be able to transfer pages at high rate, store it into the info expeditiously and additionally crawl page space. During this paper we tend to gift taxonomy of WebCrawlers.
\end{abstract}

Keywords: Crawler, Database, Search Engine, World Wide Web.

\section{INTRODUCTION}

A web crawler or spider could be a computer virus that browses the World Wide Web in sequencing and automatic manner. A crawler that is typically mentioned spider, larva or agent is computer code whose purpose it's performed net crawl. The essential design of net crawler is given below. Quite thirteen of the traffic to web site is generated by web search. these days the dimensions of the online is thousands of scores of web content that's too high and also the rate of web content are too high i.e. increasing exponentially thanks to this the most downside for computer programed is deal this quantity of the dimensions of the online. Thanks to this massive size of net induces low cowl age and computer programmer categorization not cover one third of the publically obtainable net. By analyzing varied log files of various computing device they found that most net request is generated by net crawler and it's on a median five hundredth. Crawl the online isn't a programming task, however associate formula style and system style challenge owing to the online content is incredibly massive. At present, solely Google claims to own indexed over three billion web content. The online has doubled each 9-12 months and also the dynamic rate is incredibly high. regarding four-hundredthweb content modification weekly once we take into account gently modification, however once we dynamic by one third or quite the dynamic rate is about seven-membered weekly. Researchers square measure developing new programming policy for downloading pages for the planet wide net that guarantees that, although we wish don't transfer all we tend pages we still transfer the foremost necessary (by the user purpose of view) ones.

\section{RELATED WORK}

A. World Wide Web Wanderer

In late 1993 and early 1994, when the Web was small, limited primarily to research and educational institutions, Matthew Gray implemented the World Wide Web Wanderer [2, 20]. It was written in Perl and was able to indexed pages from around 6000 sites. However, as the size of the Web increased, this crawler faced four major problems: fault tolerance, scale, politeness, and supervision. Among all serious of these problems was fault tolerance. Although the system was basically reliable, the machine running the crawler would occasionally crash and corrupt the database.

\section{B. Lycos Crawler}

Another crawler named Lycos [3, 20] was developed that ran on a single machine and used Perl's associative arrays to maintain the set of URLs to crawl. It was capable to index tens of millions of pages; however, the design of this crawler remains undocumented.

\section{Internet Archive Crawler}

Around 1997, Mike Burner's developed Internet Archive crawler [4, 20] that used multiple machines to crawl the web. Each crawler process was assigned up to 64 sites to crawl and no sites are assigned to more than one crawler. Each crawler process (single-threaded) read a list of seed URLs for its assigned sites from disk into per-site queues, and then used asynchronous I/O instructions to fetch pages from these queues in parallel. Once a page gets downloaded, the crawler extracted all the links contained in it. If a link referred to any site of the page was contained in it, then it was 


\section{IJARCCE}

added to the appropriate site queue; else it was logged to disk. Periodically, these logged "cross-site" URLs was merged by a batch process into the site-specific seed sets, filtering out duplicates one.

D. Mercator Crawler

Mercator was highly scalable and easily extensible crawler. It was written in Java. The first version [6] was nondistributed; a later distributed version [7] partitioned the URL space over the crawlers according to host name, and avoided the potential bottleneck of a centralized URL server.

\section{PROPOSED SYSTEM}

Web crawlers recursively traverse and down load net pages (the usage of GET and POST instructions) for engines like google to create and keep the net indices. The need for maintaining the as up to-date pages causes a crawler to revisit the web sites again and again. A crawler that's from time to time mentioned spider, bot or agent is software whose reason, it has done web crawling. This could be used for accessing the net pages from the internet server as in step with person bypass queries commonly for seek engine . A web crawler also used sitemap protocol for crawling net pages. In the crawling technique, normally starts with a fixed of uniform useful resource locator (urls) known as the seed url. In standard, it starts with a list of urls to visit, called seed urls. Because the crawler traverses these urls, it identifies all links inside the web page and adds them to the list of urls to be visited, referred to as the crawl frontier. Urls from the crawl frontier are visited separately and looking of the enter sample is accomplished every time text content is extracted from the web page source of the internet web page.

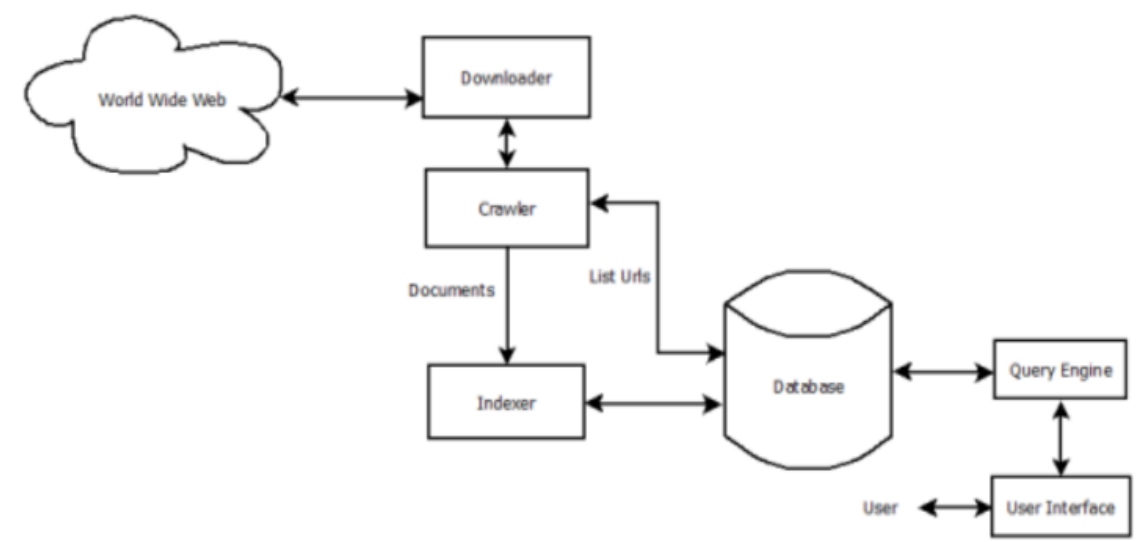

Fig. Architecture diagram

\section{IMPLEMENTATION}

We are implementing smart web crawler. All software are installed as per project requirement. Home page of application is shown as follow.

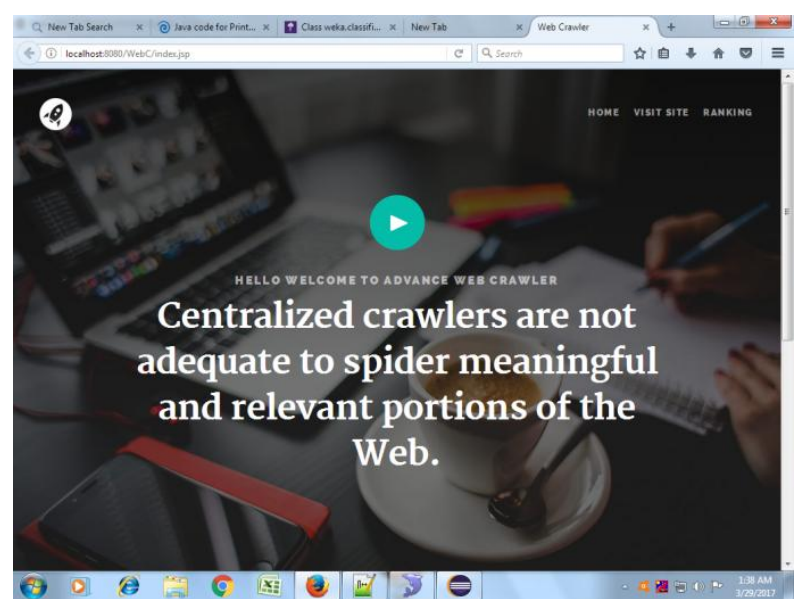

After home page, search page is displayed. Search box is given to search data. User will enter keyword to be searched as shown in fig 1 (a) and 1 (b). 


\section{IJARCCE}

International Journal of Advanced Research in Computer and Communication Engineering ISO 3297:2007 Certified

Vol. 6, Issue 5, May 2017

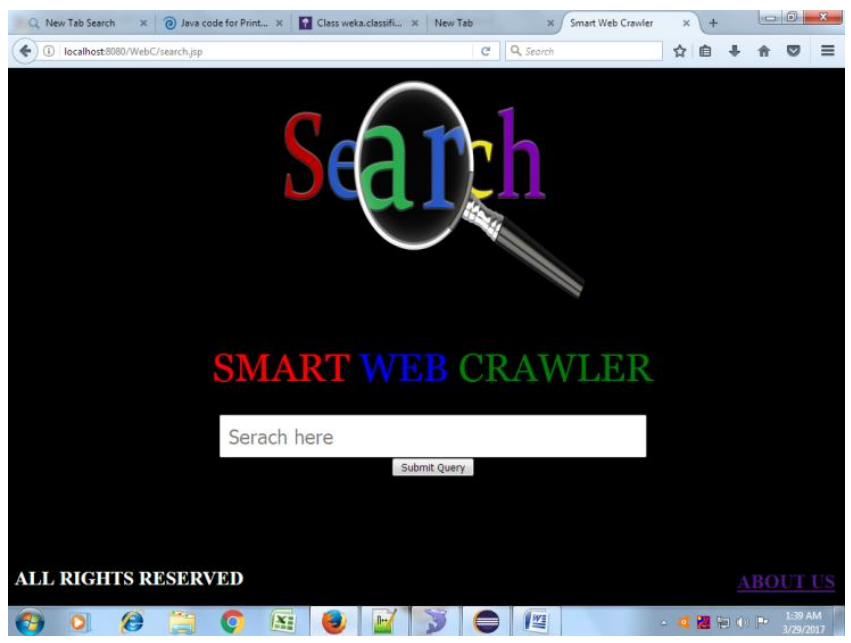

Fig 1(a)

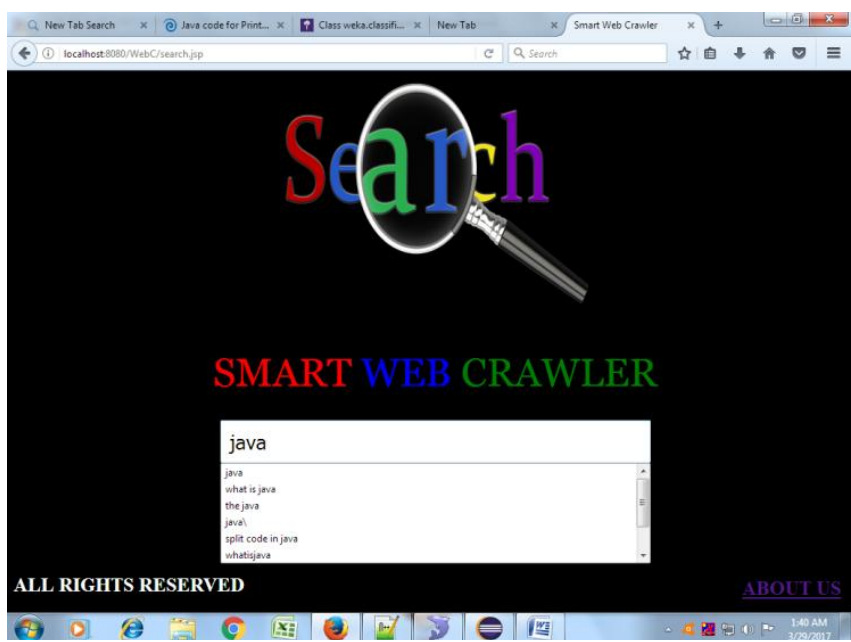

Fig 1(b)

After searching particular keyword, link will be displayed which are fetched from Google using custom search API. Three option will be displayed. We can view visited sites and ranking of each site as shown in fig 2.

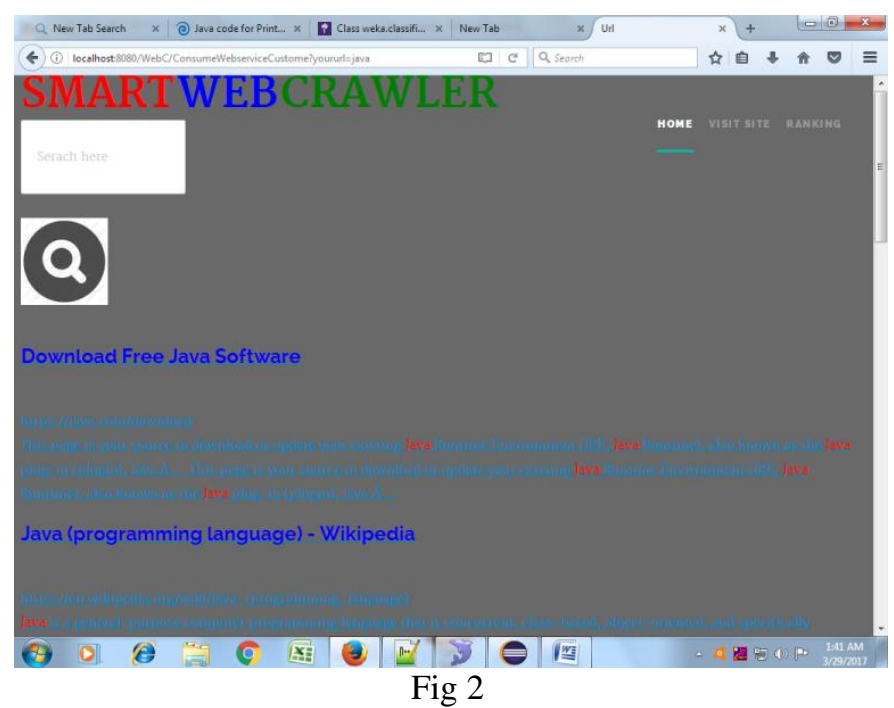

After selecting first link, Relevant web page is opened. Similarly, by selecting any link, corresponding web page is opened. It is shown in fig 3. 


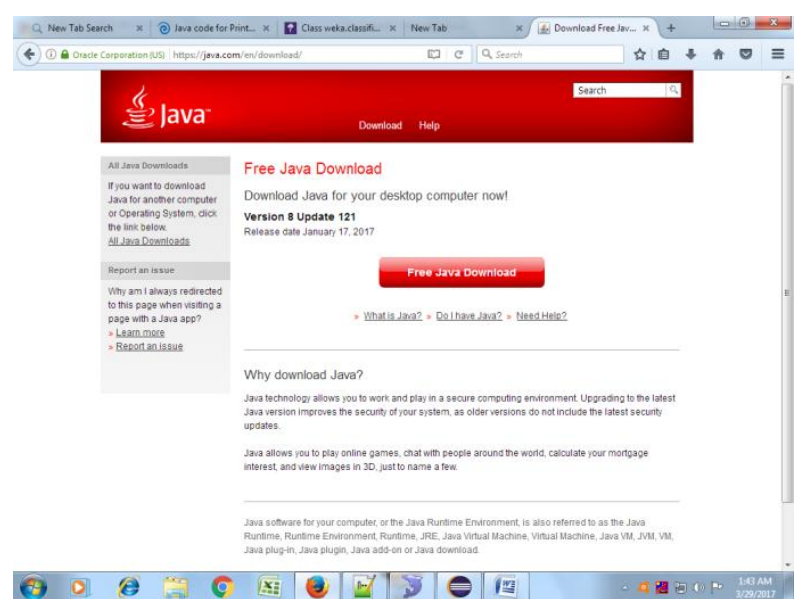

Fig 3

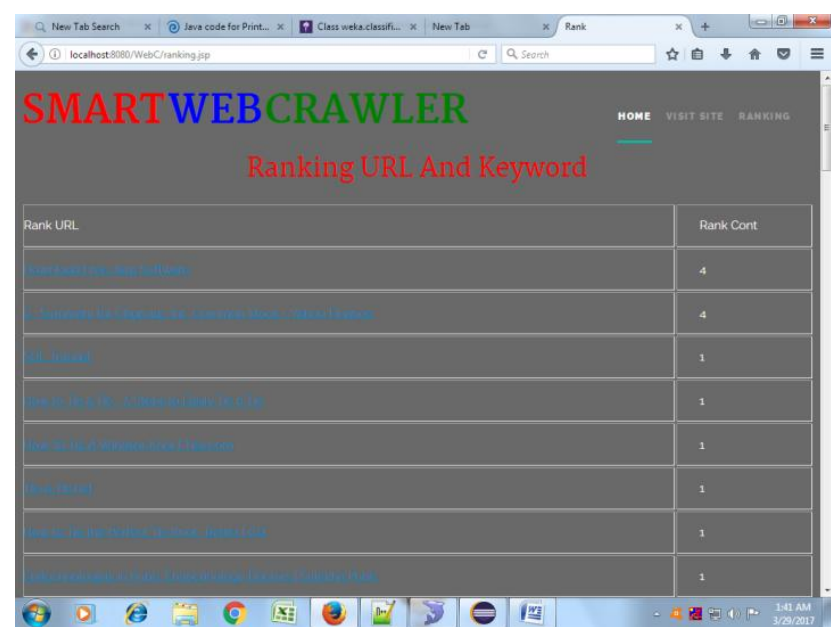

Fig. 4

Here ranking algorithm is deployed. Using this algorithm, frequency of visited sites can be calculated. We can view ranking given to each web site as shown in fig 4.

\section{RESULT}

We consider ACHE, which is an adaptive crawler for harvesting hidden-web entries with offline-online learning link classifiers. We also consider system similar to SmartCrawler, named SCDI, which shares the same stopping criteria with SmartCrawler. And we implement our proposed system. Searching through these three crawler are done for various keywords. Comparison is done and shown in graphical form.

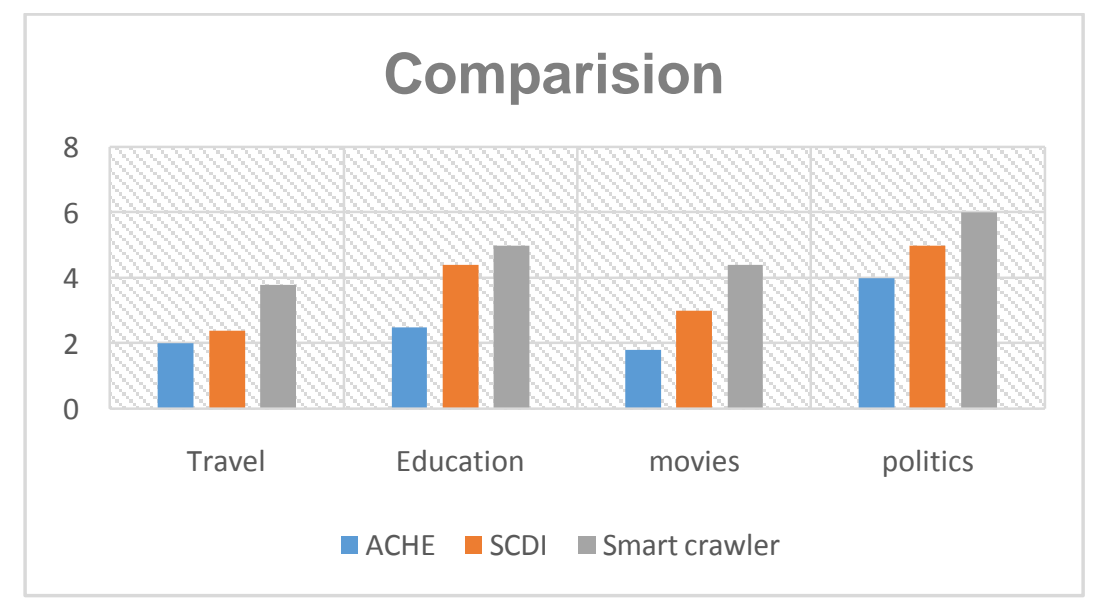




\section{CONCLUSION}

Size of the net is growing and from user purpose of read, user expects the crawler to retrieve the results as shortly as doable. owing to the dynamic manner of the net, the arrangement of pertinent pages for any given question is to bootdeeply powerful, prompting a quantifiability issue is that the supposition of a particular and complete static image of the net separates with its rate of progress. As search engines fail to meet the user's requirement for complete and recently updated data, it seems to be deeply enticing to utilize distributed net crawlers.

\section{REFERENCES}

[1] S. Abiteboul, M. Preda, and G. Cobena, "Adaptive on-line page importance computation," in Proceedings of the 12th International World Wide Web Conference, 2003.

[2] E. Adar, J. Teevan, S. T. Dumais, and J. L. Elsas, "The web changes everything: Understanding the dynamics of web content," in Proceedings of the 2nd International Conference on Web Search and Data Mining, 2009.

[3] Advanced Triage (medical term), http://en.wikipedia.org/wiki/Triage\# Advanced triage.

[4] A. Agarwal, H. S. Koppula, K. P. Leela, K. P. Chitrapura, S. Garg, P. K. GM, C. Haty, A. Roy, and A. Sasturkar, "URL normalization for deduplication of web pages," in Proceedings of the 18th Conference on Information and Knowledge Management, 2009.

[5] C. C. Aggarwal, F. Al-Garawi, and P. S. Yu, "Intelligent crawling on the world wide web with arbitrary predicates," in Proceedings of the 10th International World Wide Web Conference, 2001.

[6] D. Ahlers and S. Boll, "Adaptive geospatially focused crawling," in Proceedings of the 18th Conference on Information and Knowledge Management, 2009.

[7] Attributor. http://www.attributor.com.

[8] R. Baeza-Yates and C. Castillo, "Crawling the infinite web," Journal of Web Engineering, vol. 6, no. 1, pp. 49-72, 2007.

[9] R. Baeza-Yates, C. Castillo, M. Marin, and A. Rodriguez, "Crawling a country: Better strategies than breadth-first for web page ordering," in Proceedings of the 14th International World Wide Web Conference, 2005.

[10] B. Bamba, L. Liu, J. Caverlee, V. Padliya, M. Srivatsa, T. Bansal, M. Palekar, J. Patrao, S. Li, and A. Singh, "DSphere: A source-centric approach to crawling, indexing and searching the world wide web," in Proceedings of the 23rd International Conference on Data Engineering, 2007.

[12] Yang Sun, Isaac G. Councill, C. Lee Giles,” The Ethicality of Web Crawlers” IEEE/WIC/ACM International Conference on Web Intelligence and Intelligent Agent Technology IEEE/WIC/ACM International Conference on Web Intelligence and Intelligent Agent Technology2010.

[13] Yang Sun, Isaac G. Councill, C. Lee Giles," The Ethicality of Web Crawlers" 2010 Advances in Vision Computing: An International Journal (AVC) Vol.1, No.2/3, September 2014 